\title{
Matrix Proteins and Proteinases Network in Human Cardiovascular Diseases Explored By Cytoscape
}

\author{
Robert Jeenchen Chen $¥, \S$, Wei-hsuan Yul \\ ‡ Cardiovascular Surgery, Taipei Tzuchi Hospital, New Taipei City, Taiwan \\ § Biochemistry and Molecular Biology, National Taiwan University College of Medicine, Taipei, Taiwan \\ | Laboratory of connective tissue and stem cell research, Department of Biochemistry \& Molecular Biology, National Taiwan \\ University College of Medicine, Taipei, Taiwan
}

\begin{abstract}
Matrix proteins and proteinases altered extracellular matrix that involves in various pathogenesis of cardiovascular diseases. Systematic review selected matrix metalloproteinase-7 (MMP7), a disintegrin and metalloproteinase-17 (ADAM17), type I collagen (COL1), syndecan (SDC), and versican (VCAN). Their network was drawn by Cytoscape and Agilent Literature Search. MMP7, ADAM17, and SDC were grouped together. VCAN and COL1 had inadequate evidence to connect their human potential networks that were more evidence in animal models. The findings may provide clues for further laboratory investigations.
\end{abstract}

\section{Keywords}

Cytoscape, matrix proteins, metalloproteinase, network, cardiac disease 


\section{Introductions}

As the extracellular matrix (ECM) plays pivotal roles in regulating cardiac structural and functional integrity (Chiao et al. 2012), any alteration of ECM by matrix proteins, proteinases, and related molecules may involve in the pathophysiological process of various cardiovascular disease. (Chiao et al. 2012, Etoh et al. 2001, Neill et al. 2015, Peterson et al. 2000, Vanhoutte et al. 2006, Zile et al. 2011) We would like to explore their network relationships in human for more laboratory workup.

\section{Methods}

Thorough systematic review was by using PubMed search. On Cytoscape 3.40 (http:// www.cytoscape.org), we used Agilent Literature Search 3.11 to explore the molecular relationships. For the extraction control, the concept lexicon was "Homo sapiens", and the interaction lexicon was set to "limited". The network was drawn with GeneMANIA Force Directed layout. We screened most of the molecules available from systematic review and selected the most relevant ones for subsequent Cytoscape analysis.

\section{Data resources}

Systematic reviews with PubMed.

Online public-domain databases used by Cytoscape.

\section{Results and discussion}

From all the known and potential molecules relating to cardiovascular disease such as cardiomyopathy and transplant allograft rejection (Dupuis et al. 2015, Huet et al. 2015, Lu et al. 2000, Odenbach et al. 2010, Rickard et al. 2012, Wang et al. 2009), we selected matrix metalloproteinase-7 (MMP7), a disintegrin and metalloproteinase-17 (ADAM17), type I collagen (COL1), syndecan (SDC), and versican (VCAN). Their relational networks showed grouping of MMP7, SDC, and ADAM17 whereas only potential associations with COL1A1, COLA2, and VCAN (Fig. 1) With laboratory evidence from knock-out mice that showed interactions of COL1, VCAN, ADAM17, SDC, and MMP7, we are performing more laboratory works such as Western blotting, immunofluorescence staining, and rPCR to analyze tissue samples from human cardiomyopathy and transplant allograft rejection for validation. 


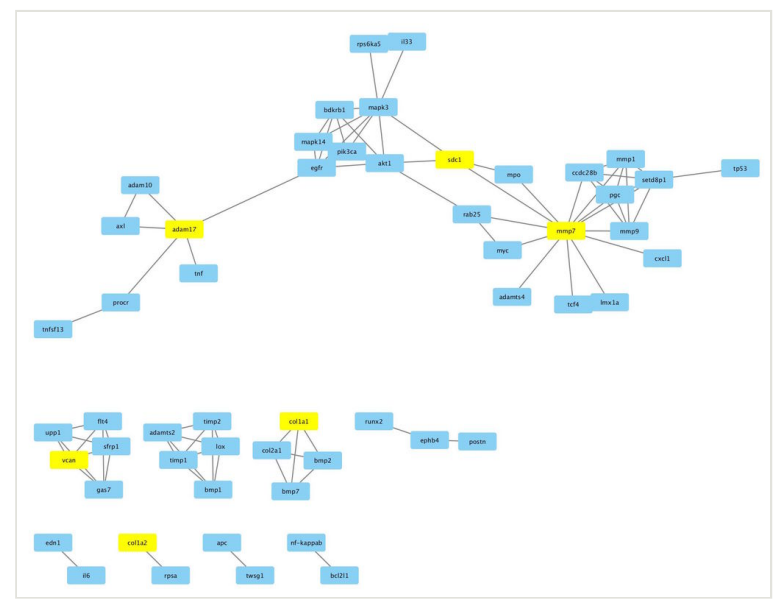

Figure 1.

Cytoscape network, derived from MMP7, ADAM17, SDC, COL1, and VCAN (see Abstract for full spellings of abbreviations).

\section{Conclusions}

Cytoscape network shows the interplaying roles of MMP7, ADAM17, SDC, COL1, and VCAN in the pathogenesis of cardiovascular diseases like cardiomyopathy and transplant rejection have been implied by the Cytoscape exploration. Further human laboratory experiments will be done to investigate the theoretical network.

\section{Presented at}

American Heart Association's Scientific Sessions 2016, November 12-16, New Orleans, Louisiana.

\section{Hosting institution}

National Taiwan University College of Medicine, Taipei, Taiwan

\section{Ethics and security}

Waived Institutional Review Board (IRB) review. 


\section{Author contributions}

Chen RJC: concept, study design, analysis, and manuscripting; Yu WH: concept, revision, and supersion.

\section{References}

- $\quad$ Chiao YA, Ramirez TA, Zamilpa R, Okoronkwo SM, Dai Q, Zhang J, Jin YF, Lindsey ML (2012) Matrix metalloproteinase-9 deletion attenuates myocardial fibrosis and diastolic dysfunction in ageing mice. Cardiovascular Research 96 (3): 444-455. [In English]. DOI: $10.1093 /$ cvr/cvs 275

- Dupuis LE, Berger MG, Feldman S, Doucette L, Fowlkes V, Chakravarti S, Thibaudeau S, Alcala NE, Bradshaw AD, Kern CB (2015) Lumican deficiency results in cardiomyocyte hypertrophy with altered collagen assembly. Journal of Molecular and Cellular Cardiology 84: 70-80. [In English]. DOI: 10.1016/i.yimcc.2015.04.007

- $\quad$ Etoh T, Joffs C, Deschamps AM, Davis J, Dowdy K, Hendrick J, Baicu S, Mukherjee R, Manhaini M, Spinale FG (2001) Myocardial and interstitial matrix metalloproteinase activity after acute myocardial infarction in pigs. American journal of physiology. Heart and circulatory physiology 281 (3): 987-94. [In English]. URL: http:// eutils.ncbi.nlm.nih.gov/entrez/eutils/elink.fcgi? $\underline{\text { dbfrom }=\text { pubmed } \& i d=11514263 \& \text { retmode }=\text { ref } \& \mathrm{cmd}=\text { prlinks }}$

- Huet E, Gabison E, Vallee B, Mougenot N, Linguet G, Riou B, Jarosz C, Menashi S, Besse S (2015) Deletion of extracellular matrix metalloproteinase inducer/CD147 induces altered cardiac extracellular matrix remodeling in aging mice. Journal of physiology and pharmacology : an official journal of the Polish Physiological Society 66 (3): 355-366. [In English]. URL: http://eutils.ncbi.nlm.nih.gov/entrez/eutils/elink.fcgi? dbfrom=pubmed \&id=26084217\&retmode $=$ ref\&cmd=prlinks

- $\quad$ Lu L, Gunja-Smith Z, Woessner JF, Ursell PC, Nissen T, Galardy RE, Xu Y, Zhu P, Schwartz GG (2000) Matrix metalloproteinases and collagen ultrastructure in moderate myocardial ischemia and reperfusion in vivo. American journal of physiology. Heart and circulatory physiology 279 (2): 601-9. [In English]. URL: http://eutils.ncbi.nlm.nih.gov/ entrez/eutils/elink.fcgi? dbfrom=pubmed\&id=10924059\&retmode $=$ ref\&cmd=prlinks

- $\quad$ Neill T, Schaefer L, lozzo RV (2015) Decoding the Matrix: Instructive Roles of Proteoglycan Receptors. Biochemistry 54 (30): 4583-4598. [In English]. DOI: 10.1021/ acs.biochem.5b00653

- $\quad$ Odenbach J, Wang X, Cooper S, Chow FL, Oka T, Lopaschuk G, Kassiri Z, FernandezPatron C (2010) MMP-2 Mediates Angiotensin II-Induced Hypertension Under the Transcriptional Control of MMP-7 and TACE. Hypertension 57 (1): 123-130. [In English]. DOI: 10.1161/HYPERTENSIONAHA.110.159525

- $\quad$ Peterson JT, Li H, Dillon L, Bryant JW (2000) Evolution of matrix metalloprotease and tissue inhibitor expression during heart failure progression in the infarcted rat. Cardiovascular Research 46 (2): 307-315. [In English]. URL: http:// eutils.ncbi.nlm.nih.gov/entrez/eutils/elink.fcgi? dbfrom $=$ pubmed $\&$ id $=10773235 \&$ retmode $=$ ref \& $\mathrm{cmd}=$ prlinks 
- $\quad$ Rickard AJ, Morgan J, Bienvenu LA, Fletcher EK, Cranston GA, Shen JZ, Reichelt ME, Delbridge LM, Young MJ (2012) Cardiomyocyte mineralocorticoid receptors are essential for deoxycorticosterone/salt-mediated inflammation and cardiac fibrosis. Hypertension 60 (6): 1443-1450. [In English]. DOI: $\underline{10.1161 /}$ HYPERTENSIONAHA.112.203158

- Vanhoutte D, Schellings M, Pinto Y, Heymans S (2006) Relevance of matrix metalloproteinases and their inhibitors after myocardial infarction: a temporal and spatial window. Cardiovascular Research 69 (3): 604-613. [In English]. DOI: 10.1016/ j.cardiores.2005.10.002

- Wang X, Chow FL, Oka T, Hao L, Lopez-Campistrous A, Kelly S, Cooper S, Odenbach J, Finegan BA, Schulz R, Kassiri Z, Lopaschuk GD, Fernandez-Patron C (2009) Matrix Metalloproteinase-7 and ADAM-12 (a Disintegrin and Metalloproteinase-12) Define a Signaling Axis in Agonist-Induced Hypertension and Cardiac Hypertrophy. Circulation 119 (18): 2480-2489. [In English]. DOI: 10.1161/CIRCULATIONAHA.108.835488

- Zile MR, DeSantis SM, Baicu CF, Stroud RE, Thompson SB, McClure CD, Mehurg SM, Spinale FG (2011) Plasma Biomarkers That Reflect Determinants of Matrix Composition Identify the Presence of Left Ventricular Hypertrophy and Diastolic Heart Failure. Circulation: Heart Failure 4 (3): 246-256. [In English]. DOI: 10.1161/ CIRCHEARTFAILURE.110.958199 